\author{
倍硫磷的甲基在分子内和分子间迁移的质谱研究 \\ 任响 ${ }^{a}$ 张小平 ${ }^{b}$ 王雨芬 ${ }^{a}$ 曹静瑜 ${ }^{a}$ 程媛媛 ${ }^{b}$ \\ 冯守华 $*, a$ 陈焕文 $*, b$ \\ ( ${ }^{a}$ 吉林大学 无机合成与制备化学国家重点实验室 长春 130012) \\ ( ${ }^{b}$ 东华理工大学 江西省质谱科学与仪器重点实验室 南昌 330013)
}

\begin{abstract}
摘要 甲基迁移反应在合成化学和生命科学领域具有重要意义. 迄今为止, 所报道的甲基迁移反应大多数发生在不同 种类分子之间. 因此，寻找新型甲基迁移反应具有一定的研究价值. 本研究以环境中常见的杀虫剂倍硫磷为研究对象, 采用电喷雾质谱技术为分析工具对反应体系进行高灵敏分析，探究在 $\mathrm{CF}_{3} \mathrm{COOH}$ 及纳米二氧化钛 $\left(\mathrm{TiO}_{2}\right)$ 条件下，倍硫磷 分子中发生甲基迁移反应的可能性. 结果表明, 在 $\mathrm{CF}_{3} \mathrm{COOH}$ 和 $\mathrm{TiO}_{2}$ 共同作用下，倍硫磷分子中的甲基可以从同一分 子的氧原子上迁移到不饱和硫原子上，发生分子内 1,3-甲基迁移反应，形成异构体硫甲基化产物；同时，异构体产物中 的甲基还可以从硫原子上再继续迁移到另一分子倍硫磷的不饱和硫原子上, 发生分子间甲基迁移反应. 同时结合密度 泛函理论计算对倍硫磷分子内和分子间甲基迁移反应的过程进行动态模拟, 并对其迁移机理进行解释. 本研究发现了 倍硫磷能发生分子内和分子间甲基迁移反应, 提供了一种研究甲基迁移反应的质谱方法, 为倍硫磷的降解研究提供了 新思路。
\end{abstract}

关键词 倍硫磷; 分子内甲基迁移; 分子间甲基迁移; 电喷雾质谱; 三氟乙酸; 纳米二氧化钠

\title{
Intramolecular and Intermolecular Methyl Migration of Fenthion Studied by Electrospray Ionization Mass Spectrometry
}

\author{
Ren, Xiang ${ }^{a}$ \\ Zhang, Xiaoping ${ }^{b}$ \\ Wang, Yufen ${ }^{a}$ \\ Cao, Jingyu ${ }^{a}$ \\ Feng, Shouhua*,a \\ Chen, Huanwen*,b \\ Cheng, Yuanyuan ${ }^{b}$ \\ ( ${ }^{a}$ State Key Laboratory of Inorganic Synthesis and Preparative Chemistry, Jilin University, Changchun 130012) \\ ( ${ }^{b}$ Jiangxi Key Laboratory for Mass Spectrometry and Instrumentation, East China Institute of Technology, Nanchang
} 330013)

\begin{abstract}
Methyl transfer reactions are of great significance in the field of synthetic chemistry and life sciences. So far, most of the reported methyl migration reactions have occurred between different types of molecules. Therefore, it is of certain value to search for new methyl transfer reactions. In this study, fenthion, a most common insecticide in the environment, was selected as the studied object, and electrospray ionization mass spectrometry (ESI-MS) was used as the analytical tool to conduct highly sensitive analysis of the reaction system, so as to explore the possibility of methyl transfer reaction in fenthion molecules under the condition of trifluoroacetic acid and nanometer titanium dioxide. Other than $\mathrm{m} / \mathrm{z} 279$ (protonated fenthion), some new product ions ( $\mathrm{m} / \mathrm{z} 293$ and $\mathrm{m} / \mathrm{z} 265)$ could be observed in the fingerprint MS of fenthion reaction solution. Tandem MS experiments showed that the intensity of product ion $\mathrm{m} / z 231$ (elimination of $\mathrm{CH}_{3} \mathrm{SH}$ ) in the dissociation of $\mathrm{m} / \mathrm{z}$ 279 from fenthion reaction solution were different from that from protonated fenthion standard. This indicated that the methyl in the fenthion could transfer from oxygen atom to unsaturated sulfur atom via 1,3-methyl transfer, forming isomer a2, which led to the high intensity of product ion $\mathrm{m} / \mathrm{z} 231$ in the dissociation of $\mathrm{m} / \mathrm{z} 279$ from fenthion reaction solution. Under the assistance of acid, the methyl cation continued to transfer from sulfur atom in $\mathbf{a} 2$ to the unsaturated sulfur atom in another fenthion molecule, forming $\mathbf{a 3}(\mathrm{m} / \mathrm{z} 293)$ and a4 via intermolecular methyl transfer reaction, which was verified by tandem MS experiments of ions at $\mathrm{m} / \mathrm{z} 293$ and $\mathrm{m} / \mathrm{z} 265$. In addition, density functional theory (DFT) calculations were carried out to confirm the mechanism of intramolecular and intermolecular methyl transfer reactions of fenthion. In order to observe the phenomenon of methyl transfer more intuitively, the effects of different acids, metal oxides, reaction time and reaction temperature on the signal intensities of ions at $\mathrm{m} / \mathrm{z} 265$ and $\mathrm{m} / \mathrm{z} 293$ of intermolecular methyl transfer reactions of fenthion were investigated. It could be concluded that under the conditions of trifluoroacetic acid and nanometer titanium dioxide, and $60{ }^{\circ} \mathrm{C}$ ultrasound reaction for $6 \mathrm{~h}$, the proportion of intermolecular methyl transfer reactions of fenthion was the highest. In
\end{abstract}

*E-mail:shfeng@jlu.edu.cn; chw8868@gmail.com

Received December 17, 2018; published February 25, 2019.

Project supported by the National Natural Science Foundation of China (No. 21427802), the National Natural Science Foundation of China (No. 21520102007, 21605017), Project of Jiangxi Provincial Department of Education (No. GJJ160574), and the Jiangxi Key Laboratory for Mass Spectrometry and Instrumentation Open Fund (JXMS201803).

项目受国家自然科学基金(No. 21427802), 国家自然科学基金(No. 21520102007, 21605017), 江西省教育厅基金(No. GJJ160574), 江西省质谱科学与 仪器重点实验室开放基金(JXMS201803)资助. 
this study, intramolecular and intermolecular methyl transfer reactions were both discovered and investigated in fenthion, which can not only provide a method to study methyl transfer reactions, but also propose a new idea for the study of degradation of fenthion.

Keywords fenthion; intramolecular methyl transfer; intermolecular methyl transfer; electrospray ionization mass spectrometry; trifluoroacetic acid; nanometer titanium dioxide

\section{1 引言}

甲基迁移反应，包括分子内甲基迁移和分子间甲基 迁移, 广泛地存在于有机合成化学和生命分析化学领 域 ${ }^{[1-4]}$, 主要应用于构建新的化学键或调控基因表达 等 $^{[5 \sim 8]}$. 大部分甲基迁移反应研究集中在不同种类分子 之间. 如半胱氨酸向蛋氨酸的转化, 化学受体中赖氨酸 残基的甲基化, DNA 的甲基化等 ${ }^{[9 \sim 11]}$. Wolfenden 等 ${ }^{[12]}$ 报道了在水溶液中甲基可以在不同的脂肪胺之间发生 迁移反应. 郭寅龙课题组 ${ }^{[13]}$ 在研究质子化 $N, N$-二甲基 异丙基苯胺与其他中性脂肪胺在气相中的反应时, 发现 甲基在两个不同分子间发生了迁移反应. 此外, 甲基迁 移反应也可发生在分子内部 ${ }^{[14 ~ 16]}$. Reepmeyer 课题组 ${ }^{[17]}$ 报道了西地那非类物质存在分子内甲基迁移反应，依据 空间有利构型, 甲基可从氮原子迁移到炭基氧原子. 赵 玉芬课题组 ${ }^{[18]}$ 对一系列氨基酸甲酯磷酰胺衍生物进行 研究, 发现在金属离子的辅助下, 氨基酸酯残基的甲基 可以在分子内向磷酸基迁移. 常见的频哪醇重排反应也 属于典型的分子内甲基迁移反应 ${ }^{[19]}$. 大多数分子内甲 基迁移的特点是甲基从一个杂原子迁移到另一个杂原 子上. 然而, 尚未发现同一化合物分子既能发生分子内 甲基迁移又能发生分子间甲基迁移的现象. 因此, 寻找 并研究新型甲基迁移反应具有重要的意义.

倍硫磷是一种广谱、速效、低毒、残效期长的有机 磷杀虫剂, 主要起触杀的胃毒作用, 渗透性较强, 适用 于各种作物、家畜等 ${ }^{[20,21]}$. 其中毒症状出现较迟, 但持 续时间长. 有研究表明, 倍硫磷的毒性作用与乙酰胆碱 酯酶(AChE)的抑制有关, 因此倍硫磷的残留具有一定 的危害 ${ }^{[22 ~ 24]}$. 倍硫磷中的 $S=P$ 官能团, 具有重要的化 学和生物性质, 在药物化学、分子生物学和农业化学等 领域具有广泛的应用价值 ${ }^{[25,26]}$. 以往的研究主要集中于 倍硫磷的定性和定量分析, 如 Wilkins 课题组 ${ }^{[27]}$ 采用 $\mathrm{AChE}$ 生物传感器分析有机溶剂中的倍硫磷, 黄贤达课 题组 ${ }^{[28]}$ 建立了液相微萃取一气相色谱-质谱联用方法测 定水样中的倍硫磷, Salm 等 ${ }^{[29]}$ 采用液相色谱一质谱法定 量测定人血中的倍硫磷等. 然而关于倍硫磷的相关反应 机理研究较少.

质谱仪作为一种快速灵敏的分析仪器, 可以提供气 相反应所需要的真空条件, 在研究反应机理方面具有独 特的优势 ${ }^{[30}$ - 33], 是深入研究有机化学反应机理的重要 工具. 结合碰撞诱导解离多级串联质谱技术, 可更快捷 的获取中间体离子或产物离子的结构信息. 本文以倍硫 磷为研究对象, 采用 $\mathrm{CF}_{3} \mathrm{COOH}$ 与纳米二氧化钛 $\left(\mathrm{TiO}_{2}\right)$ 协同作用策略, 探究倍硫磷分子内与分子间的甲基迁移
反应，并应用电喷雾质谱(ESI-MS)技术对甲基迁移反应 过程进行监测, 结合密度泛函理论计算对倍硫磷分子内 和分子间甲基迁移的反应过程进行动态模拟, 并对其迁 移机理进行了解释. 本研究发现了倍硫磷能发生分子内 和分子间甲基迁移反应，提供了一种研究甲基迁移反应 的质谱方法, 同时有望为倍硫磷的降解研究提供新思 路.

\section{2 结果与讨论}

\section{1 倍硫磷的一级质谱}

图 1a 为倍硫磷标准品的高分辨一级化学指纹谱图. 从图中可观察到信号较强的质子化离子 $\mathrm{m} / \mathrm{z}$ $279.02703\left([\mathrm{M}+\mathrm{H}]^{+}\right)$和钠合离子 $m / z \quad 301.00891([\mathrm{M}+$ $\mathrm{Na}]^{+}$). 与对应离子质荷比的理论值非常一致, 误差在 2 ppm 以内.
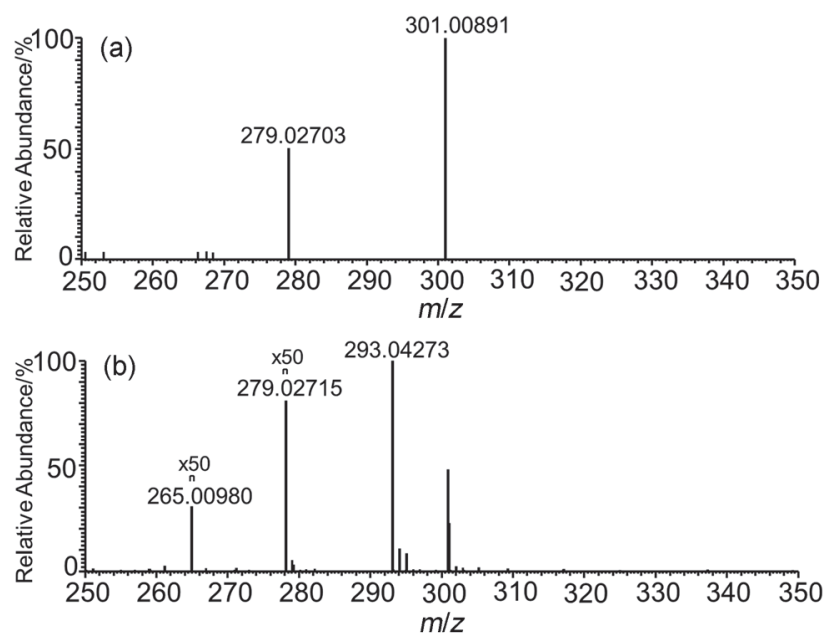

图 1 高分辨一级质谱图. (a) 倍硫磷标准品; (b) 倍硫磷在 $\mathrm{CF}_{3} \mathrm{COOH}$ 和 $\mathrm{TiO}_{2}$ 条件下反应 $6 \mathrm{~h}$ 后的反应液

Figure 1 High resolution MS. (a) fenthion standard; (b) Resultant of the authentic fenthion incubated with $\mathrm{CF}_{3} \mathrm{COOH}$ and $\mathrm{TiO}_{2}$ for $6 \mathrm{~h}$

\section{2 倍硫磷分子内甲基迁移}

图 2a 是以倍硫磷标准品分子(a1)质子化的 $m / z 279$ 为母离子, 进行碰撞诱导解离(CID)实验的二级质谱图, 可观测到基峰碎片离子 $m / z 247$ 和两个低丰度碎片离子 $m / z 231$ 和 $m / z 169$, 分别为母离子丢失 $\mathrm{CH}_{3} \mathrm{OH} 、 \mathrm{CH}_{3} \mathrm{SH}$ 和 $\mathrm{C}_{2} \mathrm{H}_{7} \mathrm{O}_{3} \mathrm{P}$ 所致. 其中, 碎片离子 $m / z 169$ 的产生涉及到 硫的迁移重排反应，这在我们之前的工作中已有详细研 究. 碎片离子 $m / z 247$ 和 231 的产生均涉及到外加质子 的迁移反应，进而分别丢失甲醇和硫甲醇，这已通过氛 
同位素标记实验进行验证, 详细见文献 ${ }^{[34]}$. 倍硫磷标准 品在 $\mathrm{CF}_{3} \mathrm{COOH}$ 与 $\mathrm{TiO}_{2}$ 协同作用下反应 $6 \mathrm{~h}$ 后, 仍然能 在其反应混合物中观测到高强度 $m / z 279.02715$ 离子(图 1b). 然而, 虽然该离子的碎片离子为 $\mathrm{m} / \mathrm{z} 247 、 \mathrm{~m} / \mathrm{z} 231$ 和 $m / z 169$, 与质子化倍硫磷的碎片离子的质荷比相同, 但是, 这些碎片离子的丰度有较大差异(图 2b). 比较图 $2 \mathrm{a}$ 和图 $2 \mathrm{~b}$ 可发现, 碎片中丰度差异最明显的是 $m / z 231$ 碎片离子, 该离子为母离子 $m / z 279$ 丢失 $\mathrm{CH}_{3} \mathrm{SH}$ 的产物. 这些差异表明图 $2 \mathrm{a}$ 与图 $2 \mathrm{~b}$ 中的母离子的质荷比虽然相 同, 但是它们丢失 $\mathrm{CH}_{3} \mathrm{SH}$ 的难易程度差别很大, 进而说 明反应混合液中观测到的信号 $m / z 279$ 并不是来自倍硫 磷本身, 推测可能是其异构化产物 a2. a2 结构中质子化 位点易结合在不饱和氧原子上, 在正离子活化与驱动 下, 外加质子可发生 1,3-迁移反应至硫原子上, 促使其 活化而发生 $\mathrm{P}-\mathrm{S}$ 断裂, 进而容易丢失硫甲醇中性小分 子. 而 $\mathrm{a} 1$ 结构中质子化位点易结合在不饱和硫原子上, 在正离子活化与驱动下, 外加质子可发生 1,3-迁移反应 至氧原子上, 促使其活化而发生 $\mathrm{P}-\mathrm{O}$ 断裂, 进而容易 丢失甲醇中性小分子. 因此, 质子化的 a2 比 a1 更容易 丢失 $\mathrm{CH}_{3} \mathrm{SH}$ 产生碎片 $\mathrm{m} / \mathrm{z} 231$.
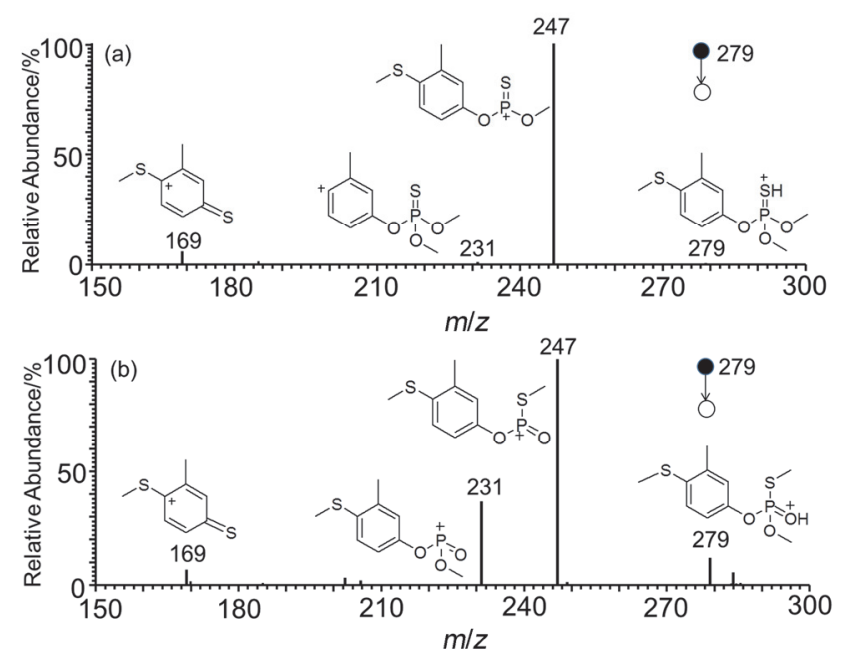

图 2 串联质谱分析 $m / z$ 279. (a) 倍硫磷标准品; (b) 倍硫磷在 $\mathrm{CF}_{3} \mathrm{COOH}$ 和 $\mathrm{TiO}_{2}$ 条件下反应 $6 \mathrm{~h}$ 后的反应液

Figure 2 ESI-MS/MS spectra of $m / z$ 279. (a) Protonated authentic fenthion standards ( $\mathrm{m} / \mathrm{z} 279)$; (b) Resultant of the authentic fenthion incubated with $\mathrm{CF}_{3} \mathrm{COOH}$ and $\mathrm{TiO}_{2}$ for $6 \mathrm{~h}$

由于反应液质谱图中 $m / z 279$ 离子非常容易丢失一 分子 $\mathrm{CH}_{3} \mathrm{SH}$, 可初步推断形成该离子的分子前体具有 $\mathrm{SCH}_{3}$ 结构. 此外, $\mathrm{P}=\mathrm{O}$ 键能约为 $585 \mathrm{~kJ} / \mathrm{mol}$; 而 $\mathrm{P}=\mathrm{O}$ 的键长比 $\mathrm{P}=\mathrm{S}$ 短, 即 $\mathrm{P}=\mathrm{S}$ 的键能比 $\mathrm{P}=\mathrm{O}$ 要低. 因此, 当分子内同时存在 $\mathrm{P}=\mathrm{S}$ 和 $\mathrm{P}-\mathrm{O}$ 时, $\mathrm{P}=\mathrm{S}$ 易断键并形成 $\mathrm{P}-\mathrm{S}$ 和 $\mathrm{P}=\mathrm{O}$, 导致倍硫磷分子 (a1)中的甲基易从氧原 子上迁移到不饱和硫原子上, 形成异构体硫甲基化产物 $\mathbf{a 2}$, 即发生分子内 1,3-甲基迁移(Scheme 1). 文献表明, Newman-Kwart 重排反应中, 芳基也很容易从氧原子上
迁移到硫原子上，该反应可以很好地用来制备苯酚 ${ }^{[35]}$.

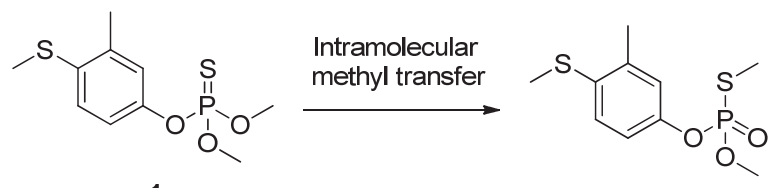

a1

a2

图式 1 倍硫磷分子内甲基迁移

Scheme 1 Intramolecular methyl transfer occurred in fenthion molecules

\section{3 倍硫磷分子间甲基迁移}

图 $1 \mathrm{~b}$ 中除观测到离子 $m / z 279.02715$ 外, 还有基峰 离子 $m / z 293.04273$ 和丰度较低的离子 $m / z 265.00980$. 其中, $m / z 293.04273$ 和 $m / z 265.00980$ 对应的分子式为 $\mathrm{C}_{11} \mathrm{H}_{18} \mathrm{O}_{3} \mathrm{PS}_{2}$ 和 $\mathrm{C}_{9} \mathrm{H}_{14} \mathrm{O}_{3} \mathrm{PS}_{2}$, 比质子化的倍硫磷分子式 多了一个亚甲基和少了一个亚甲基. 这些不同结构的化 合物大量存在，说明在 $\mathrm{CF}_{3} \mathrm{COOH}$ 和 $\mathrm{TiO}_{2}$ 协同作用下， 倍硫磷发生了分子间的甲基迁移反应. 在一级质谱图 $1 \mathrm{~b}$ 中的基峰离子 $(\mathrm{m} / \mathrm{z} 293.04273$, 即 $\mathbf{a 3})$ 易丢失甲基，得 到高强度碎片离子 $m / z 278$ (图 3a); 该离子可继续裂解, 获得碎片离子 $m / z 245$ 和低丰度碎片离子 $m / z$ 263、 $m / z$ 231(图 3b), 分别为母离子 $m / z 278$ 丢失 $\mathrm{SH}$ 自由基、 $\mathrm{CH}_{3}$ 自由基和 $\mathrm{CH}_{3} \mathrm{~S}$ 自由基后的产物(Scheme $2 \mathrm{a}$ ). 同理, 对

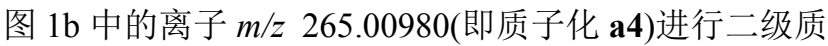
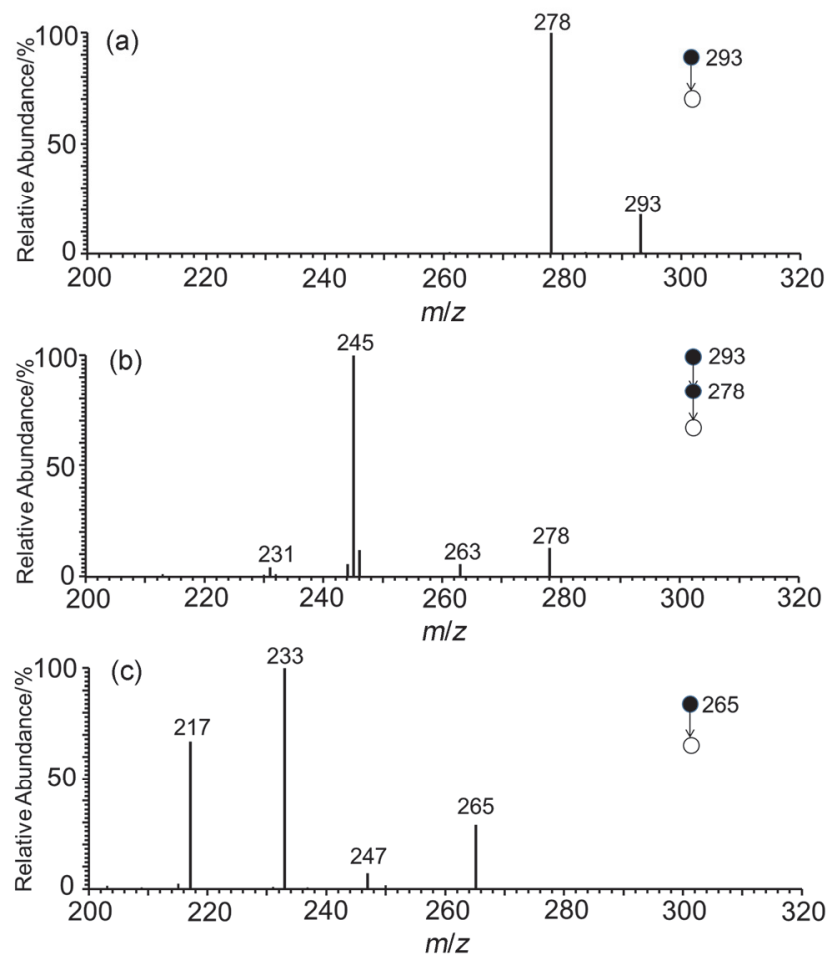

图 3 倍硫磷在 $\mathrm{CF}_{3} \mathrm{COOH}$ 和 $\mathrm{TiO}_{2}$ 条件下反应 $6 \mathrm{~h}$ 后反应液的质谱图 (a) $m / z 293$ 二级质谱图 (b) $m / z 293$ 三级质谱图 (c) $m / z 265$ 二级质谱 图

Figure 3 ESI-MS ${ }^{\mathrm{n}}$ spectra of fenthion solution with trifluoroacetic acid and titanium dioxide (a) $\mathrm{MS}^{2}$ spectrum of $m / z 293$; (b) $\mathrm{MS}^{3}$ spectrum of $m / z 293$; (c) $\mathrm{MS}^{2}$ spectrum of $m / z 265$ 
谱分析, 可获得碎片离子 $m / z 217, m / z 233$ 和 $m / z$ 247(图 $3 \mathrm{c}$ ), 分别为母离子丢失 $\mathrm{CH}_{3} \mathrm{SH} 、 \mathrm{CH}_{3} \mathrm{OH}$ 和 $\mathrm{H}_{2} \mathrm{O}$ 后的产 物, 相关过程碎裂途径见 Scheme 2b. 这些结构数据表 明，倍硫磷分子中的甲基发生分子内迁移后形成 $\mathbf{a} 2$; 在 酸等催化作用下, $\mathbf{a} 2$ 的甲基继续从硫原子上迁移到了另 一分子倍硫磷(a1)的不饱和硫原子上, 形成 $\mathbf{a} 3$ 和 $\mathbf{a 4}$, 见 Scheme 3.

\section{4 理论计算}

为进一步从分子水平上阐明相关反应的过程, 采用 DFT 方法对倍硫磷的分子内甲基迁移和分子间甲基迁 移反应过程进行了初步的计算. 图 4 为倍硫磷发生分子 内甲基迁移反应的势能面图. 从图 4 中可以看出, 倍硫 磷(a1)中氧原子上的甲基发生 1,3-迁移(过渡态, TS1)后 形成产物 $\mathbf{a} 2, \mathbf{a} 2$ 的能量比 $\mathbf{a} 1$ 低 $7.2 \mathrm{kcal} / \mathrm{mol}$, 即 $\mathbf{a} 2$ 更稳 定些. 从热力学角度表明, 反应放热 $(\Delta G<0)$, 该迁移反 应能自发进行. 质谱实验结果也说明倍硫磷容易发生 1,3-甲基迁移后形成产物 $\mathbf{a} 2$.
图 5 为倍硫磷发生分子内甲基迁移形成 $\mathbf{a} 2$ 后，在酸 的作用下，质子化 $\mathbf{a} 2$ 继续与另一分子倍硫磷发生分子 间甲基迁移反应的势能面图. 质子化 $\mathbf{a} 2$ 与 $\mathbf{a} 1$ 通过相互 作用形成 complex 1. 其能量比质子化 $\mathbf{a} 2$ 和 $\mathbf{a} 1$ 的能量之 和低 $6.2 \mathrm{kcal} / \mathrm{mol}$, 这主要是因为 $\mathbf{a} 1$ 和 $\mathbf{a} 2$ 形成了分子间 氢键 $\mathrm{O}-\mathrm{H} \cdots \mathrm{S}(2.047 \AA)$, 降低了体系的能量. 然后, $\mathbf{a} 2$ 中硫上的甲基迁移至 $\mathbf{a} 1$ 中不饱和硫原子上形成 complex 2, 该过程所需要的能垒为 $52.5 \mathrm{kcal} / \mathrm{mol}(\mathrm{TS} 2)$. 克服该能垒所需的能量可能来源于外界加热超声或酸 的催化. 其中在酸的催化下引入正电荷, 并在氢键作用 下形成稳定的 complex 1, 进一步诱导分子内甲基迁移 反应的发生, 驱动反应由 complex 1 转化为 complex 2 . 这种正电荷驱动的重排反应在质谱中也比较常见. 甲基 迁移的过程 $\mathbf{a} 2$ 中 $\mathrm{P}-\mathrm{S}$ 键长缩短(由 $2.059 \AA$ 变至 1.981 $\AA$ ), 而 $\mathbf{a 1}$ 中 $\mathrm{P}-\mathrm{S}$ 键长拉长(由 $1.940 \AA$ 变至 $1.977 \AA$ ). 随 后, complex 2 继续分解形成 $\mathbf{a 3}$ 和 $\mathbf{a 4}$, 该过程体系能量 降低 $2.8 \mathrm{kcal} / \mathrm{mol}$, 反应自发进行. 这与质谱实验观测到

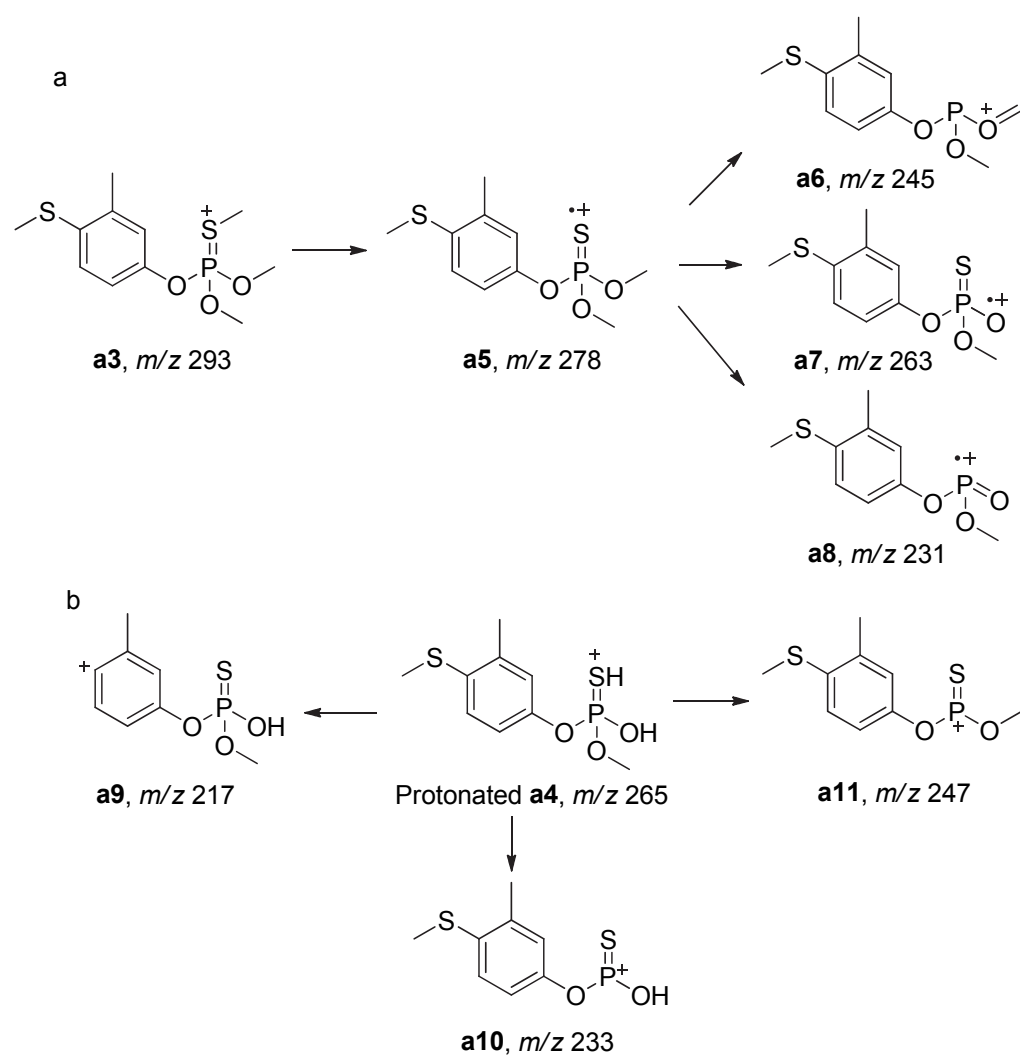

图式 $2 \mathrm{~m} / \mathrm{z} 293$ 和 $m / z 265$ 的碎裂途径

Scheme 2 The tentative fragmentation pathways of $\mathrm{m} / \mathrm{z} 293$ and $\mathrm{m} / \mathrm{z} 265$<smiles>COP(=S)(OC)Oc1ccc(SC)c(C)c1</smiles>

图式 3 分子间甲基迁移

Scheme 3 Intermolecular transfer of methyl groups 


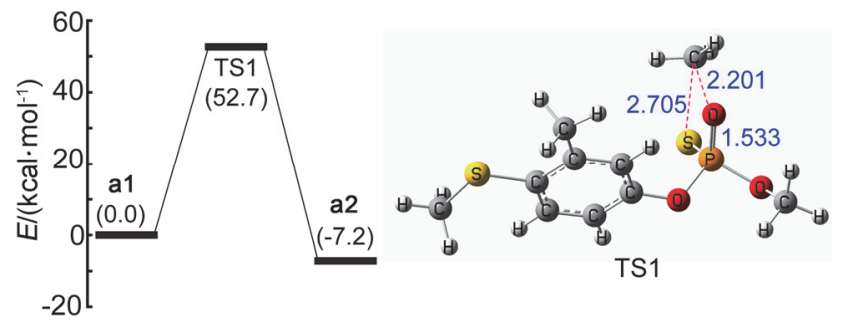

图 4 倍硫磷分子内甲基迁移中间体的结构和相对能量

Figure 4 DFT-calculated structures and relative energies for intermediates involved in intramolecular methyl migration of fenthion
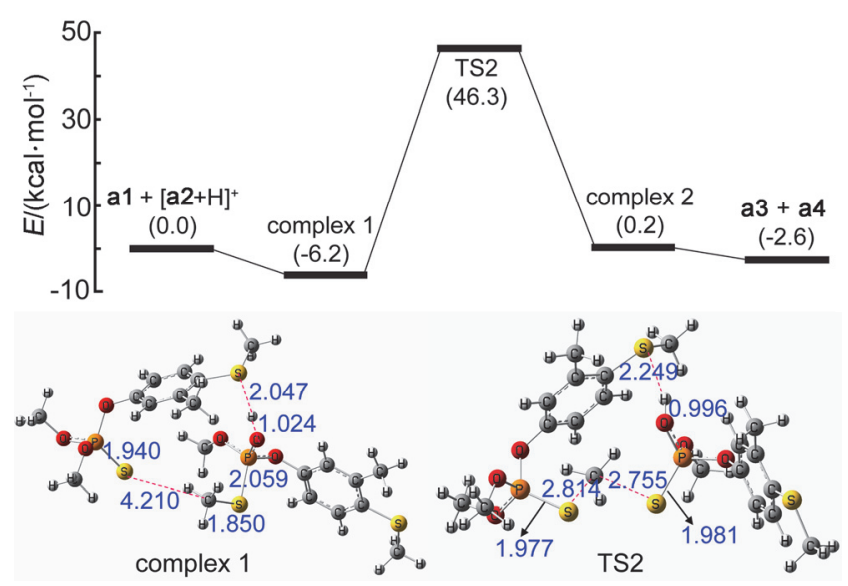

图 5 倍硫磷分子间甲基迁移中间体的结构和相对能量

Figure 5 DFT-calculated structures and relative energies for intermediates involved in intermolecular methyl migration of fenthion
的结果相一致.

\section{5 实验条件的优化}

为了考察不同的实验条件对甲基迁移反应的影响， 分别考察了不同的酸、金属氧化物、反应时间和温度对 倍硫磷甲基迁移的目标离子峰 $m / z 265$ 和 $m / z 293$ 信号强 度的影响. 在 $\mathrm{H}_{3} \mathrm{PO}_{4} 、 \mathrm{HNO}_{3} 、 \mathrm{CF}_{3} \mathrm{COOH} 、 \mathrm{H}_{2} \mathrm{SO}_{4}$ 和 $\mathrm{HCl}$ 五种酸分别与纳米 $\mathrm{TiO}_{2}$ 协同作用下, 按照 4.2 节实验操 作，探究了不同酸对倍硫磷甲基迁移反应的检测信号强 度影响. 如图 6a 所示, $\mathrm{CF}_{3} \mathrm{COOH}$ 与纳米 $\mathrm{TiO}_{2}$ 共同作用 下，可观测到信号较强的离子峰 $m / z 265$ 和 $m / z$ 293. 其 他无机酸与纳米 $\mathrm{TiO}_{2}$ 共同作用下，目标离子峰的信号 强度较弱. 这可能是由于三氟乙酸是一种有机强酸, 且 作为有机溶剂与倍硫磷具有较好的溶解性. 而其他酸虽 酸性也比较强, 但属于无机酸, 需在水溶液中电离出氢 离子, 降低了倍硫磷的溶解性. 因此, $\mathrm{CF}_{3} \mathrm{COOH}$ 和 $\mathrm{TiO}_{2}$ 可能对这个反应有催化作用，同时在正电荷的驱动下促 进甲基迁移反应的发生. 图 $6 \mathrm{~b}$ 为在 $\mathrm{CF}_{3} \mathrm{COOH}$ 酸化条件 下，不同金属氧化物对倍硫磷甲基迁移反应的影响. 从 图 $6 \mathrm{~b}$ 可知, 与非纳米类金属氧化物相比, 纳米类的金属 氧化物与酸协同催化效果较好, 可能与纳米类金属氧化 物的比表面积大有关. 其中在 $\mathrm{CF}_{3} \mathrm{COOH}$ 和纳米 $\mathrm{TiO}_{2}$ 条 件下离子峰信号强度增强, 可能是纳米 $\mathrm{TiO}_{2}$ 与 $\mathrm{CF}_{3} \mathrm{COOH}$ 强酸共同作用, 催化性能增强促进了甲基迁 移反应进行, 进而获得较高的信号强度. 由于反应温度 可促进有机反应进行同时可能导致倍硫磷发生降解反
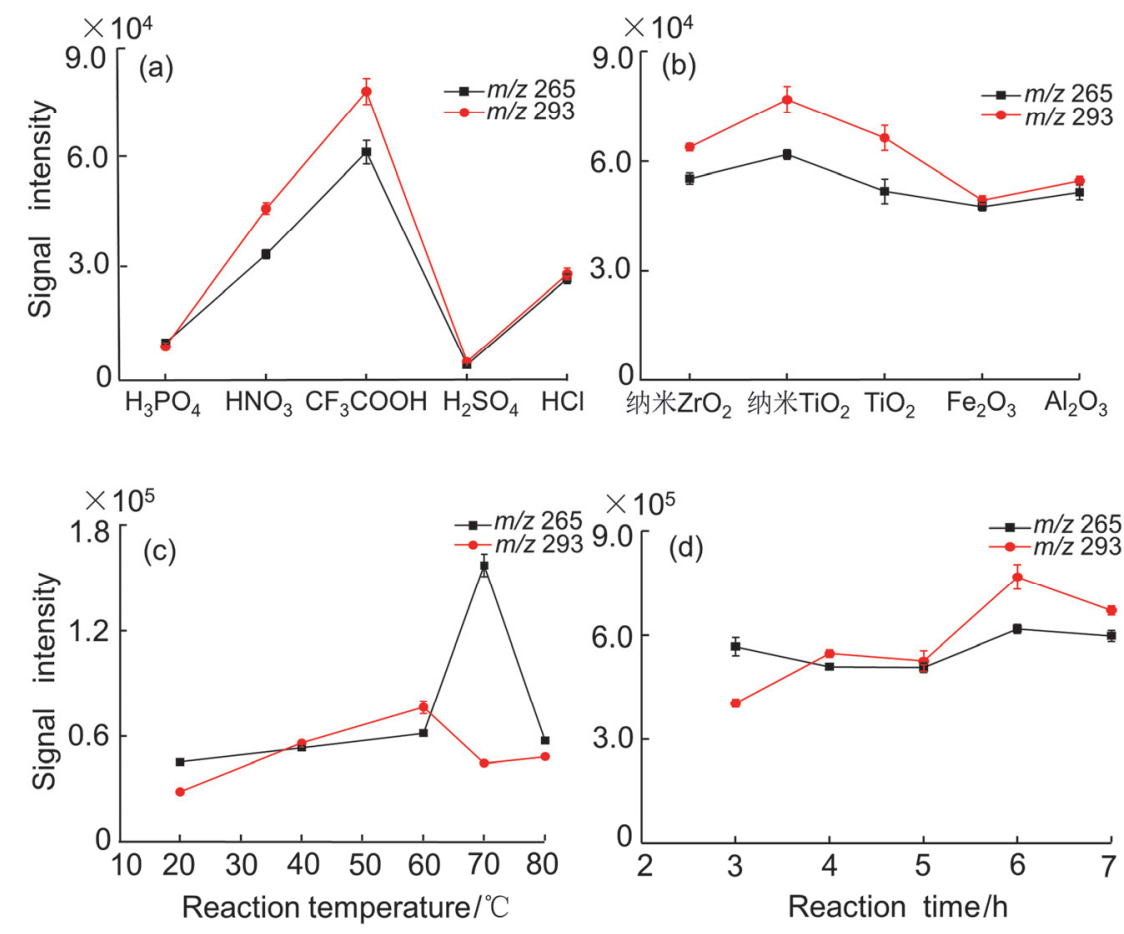

图 6 实验条件的优化: (a) 不同酸; (b) 金属氧化物; (c) 反应温度; (d) 反应时间

Figure 6 Optimization of experimental conditions: (a) Different acids; (b) Metallic oxide; (c) Reaction temperature; (d) Reaction time 
应，因此，在 $\mathrm{CF}_{3} \mathrm{COOH}$ 和纳米 $\mathrm{TiO}_{2}$ 共同作用情况下， 考察了不同反应温度对目标离子峰信号强度的影响. 如 图 6c 所示, 当温度低于 $60{ }^{\circ} \mathrm{C}$ 时, 随温度升高, 离子峰 $m / z 293$ 和 $m / z 265$ 信号强度逐渐增强; 当温度高于 $60{ }^{\circ} \mathrm{C}$ 时, $m / z 293$ 信号强度减弱; $m / z 265$ 先升高随后也 降低, 这可能是由于温度过高, 倍硫磷反应液发生了降 解反应，抑制了甲基迁移反应. 图 $6 \mathrm{~d}$ 为在 $\mathrm{CF}_{3} \mathrm{COOH}$ 和 纳米 $\mathrm{TiO}_{2}$ 协同作用, $60{ }^{\circ} \mathrm{C}$ 水浴条件下, 不同的超声时 间对倍硫磷甲基迁移反应的影响. 随着反应时间增加, 甲基迁移产物的信号强度逐渐增加，当反应时间超过 6 $\mathrm{h}$ 时, 离子峰信号强度呈下降趋势, 这可能是反应时间 过长, 倍硫磷发生了少部分的降解反应或其他有机反应 (图 6d). 综合上述条件, 可发现在 $\mathrm{CF}_{3} \mathrm{COOH}$ 和纳米 $\mathrm{TiO}_{2}$ 协同作用下, $60{ }^{\circ} \mathrm{C}$ 水浴超声反应 $6 \mathrm{~h}$ 的实验条件, 发生分子间甲基迁移反应的比例是最高的.

\section{3 结论}

本文采用电喷雾质谱 (ESI-MS) 技术, 发现在 $\mathrm{CF}_{3} \mathrm{COOH}$ 与纳米二氧化钛 $\left(\mathrm{TiO}_{2}\right)$ 协同作用策略下，倍 硫磷会发生分子内 1,3-甲基迁移反应，形成异构体硫甲 基化产物 $\mathbf{a} 2, \mathbf{a} 2$ 中的甲基继续迁移到另一分子倍硫磷的

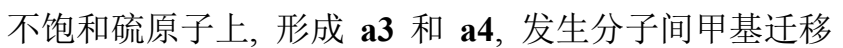
反应. 结合 DFT 计算对倍硫磷分子内和分子间甲基迁 移的机理进行解释. 本方法具有样品耗量少、操作简单 以及分析速度快等优点, 有望应用于倍硫磷降解的环境 领域研究中, 同时为探索同一分子新型甲基迁移反应提 供了一种快速质谱分析新方法.

\section{4 实验部分}

\section{1 仪器与试剂}

线性离子阱静电场轨道阱液质联用仪(LTQ Orbitrap-XL)并配有 Xcalibur 数据处理系统(美国 Thermo Scientific 公司)、KQ-300DE 型数控超声波清洗器(上 海)、倍硫磷(上海百灵威科技有限公司)、纳米 $\mathrm{TiO}_{2}$ (上 海百灵威科技有限公司)、 $\mathrm{TiO}_{2}$ (上海麦克林科技有限公 司)、纳米 $\mathrm{ZrO}_{2}$ (上海麦克林科技有限公司)、 $\mathrm{Fe}_{2} \mathrm{O}_{3}$ (上海 麦克林科技有限公司)、 $\mathrm{Al}_{2} \mathrm{O}_{3}$ (上海麦克林科技有限公 司)、 $\mathrm{CF}_{3} \mathrm{COOH}$ (上海百灵威科技有限公司)、 $\mathrm{H}_{3} \mathrm{PO}_{4}$ (上 海麦克林科技有限公司) $\mathrm{HNO}_{3}$ (上海麦克林科技有限公 司)、 $\mathrm{H}_{2} \mathrm{SO}_{4}$ (上海麦克林科技有限公司)、 $\mathrm{HCl}$ (上海麦克 林科技有限公司)、甲醇(色谱纯, 美国 ROE 公司)、二次 蒸馏水.

\section{2 实验过程}

取 $2 \mu \mathrm{L}$ 倍硫磷标准品于 $2 \mathrm{~mL}$ 反应瓶中作为反应底 物. 称取 $1 \mathrm{mg}$ 纳米二氧化钛 $\left(\mathrm{TiO}_{2}\right)$ 于反应瓶中, 再向其 中加入 $50 \mu \mathrm{L} \mathrm{CF}_{3} \mathrm{COOH}$. 将反应瓶置于 $60{ }^{\circ} \mathrm{C}$ 水浴中超 声 $6 \mathrm{~h}$. 利用 ESI-MS 装置监测反应液, 获得倍硫磷甲基
迁移反应产物的特征化学指纹谱图以及串联质谱图.

采用正离子扫描模式; 离子传输管温度为 $150{ }^{\circ} \mathrm{C}$; 质量扫描范围为 $m / z 50 \sim 500 \mathrm{Da}$ 、甲醇水 $(V / V, 3 / 7)$ 作为 萃取溶剂, 流速 $3.5 \mu \mathrm{L} / \mathrm{min}$; 毛细管电压 $3.5 \mathrm{kV}$ 、在进行 串联质谱时，母离子隔离宽度是 $1.0 \mathrm{Da}$, 设置碰撞能量 为 $10 \% \sim 30 \%$, 碰撞时间为 $30 \mathrm{~ms}$; 其它检测参数由质 谱仪系统自动优化得到.

\section{3 理论计算}

理论计算采用 Gaussian-09 程序包完成, 通过密度 泛函理论中的 B3LYP 方法进行结构优化与频率计算. 其中，反应物、过渡态、产物的分子优化构型、振动频 率均在 6-31+ $\mathrm{G}(\mathrm{d}, \mathrm{p})$ 水平上进行. 同时, 采用内禀反应 坐标(IRC)计算确认各过渡态所连接的反应物和产物. 所有结构的频率计算都在同一基组进行.

\section{References}

[1] Dakternieks, D.; Lim, A. E. K.; Lim, K. F. Chem. Commun. 1999, $15,1425$.

[2] Shekar, S.; Brown, S. N. J. Org. Chem. 2014, 79, 12047.

[3] Schmidt, T.; Schwede, T.; Meuwly, M. J. Phys. Chem. B 2014, 118, 5882.

[4] Lukinavicius, G.; Lapiene, V.; Stasevskij, Z.; Dalhoff, C.; Weinhold, E.; Klimasauskas, S. J. Am. Chem. Soc. 2007, 129, 2758.

[5] Khaskin, E.; Zavalij, P. Y.; Vedernikov, A. N. J. Am. Chem. Soc. 2008, 130, 10088

[6] Shekar, S.; Brown, S. N. Organometallics 2013, 32, 556.

[7] Jones, P. A.; Takai, D. Science 2001, 293, 1068.

[8] Klose, R. J.; Bird, A. P. Trends Biochem. Sci. 2006, 31, 89.

[9] Finnegan, E. J.; Peacock, W. J.; Dennis, E. S. Curr. Opin. Genet. Dev. 2000, 10, 217.

[10] Xiong, L.; Ping, L.; Yuan, B.; Wang, Y. J. Am. Soc. Mass Spectrom. 2009, 20, 1172 .

[11] Hughes, R. M.; Waters, M. L. J. Am. Chem. Soc. 2005, 127, 6518.

[12] Callahan, B. P.; Wolfenden, R. J. Am. Chem. Soc. 2003, 125, 310.

[13] Zhang, X.; Wang, H.; Liao, Y.; Ji, H.; Guo, Y. J. Mass Spectrom. 2010, 42, 218.

[14] Kshirsagar, U. A.; Argade, N. P. Tetrahedron 2009, 65, 5244

[15] Zhang, X. J. Mol. Struc.-Theochem. 2010, 955, 91.

[16] Zhang, X.; Yao, S.; Guo, Y. Int. J. Mass Spectrom. 2008, 270, 31

[17] Reepmeyer, J. C. Rapid Commun. Mass Spectrom. 2010, 23, 927.

[18] Gao, X.; Zhu, G.; Zeng, Z.; Chen, W.; Lin, Z.; Liu, Y.; Xu, P.; Zhao, Y. Rapid Commun. Mass Spectrom. 2011, 25, 1061.

[19] Ammal, S. C.; Yamataka, H.; Aida, M. A.; Dupuis, M. Science 2003, 299, 1555.

[20] Kitamura, S.; Kadota, T.; Yoshida, M.; Jinno, N.; Ohta, S. Comp. Biochem. Phys. C 2000, 126, 259.

[21] Bai, C. L.; Qiao, C. B.; Zhang, W. D.; Chen, Y. L.; Qu, S. X. Biomed. Environ. Sci. 1990, 3, 262

[22] Ben Amara, I.; Sefi, M.; Troudi, A.; Soudani, N.; Boudawara, T.; Zeghal, N. Indian J. Biochem. Bio. 2014, 51, 293.

[23] Cheke, R. A.; Mcwilliam, A. N.; Mbereki, C.; Van Der Walt, E.; Mtobesya, B.; Magoma, R. N.; Young, S.; Eberly, J. P. Ecotoxicology 2012, 21, 1761.

[24] Celik, I.; Isik, I.; Ozok, N.; Kaya, M. S. Toxicol. Ind. Health 2011, $27,357$.

[25] Eckstein, F.; Gish, G. Trends Biochem. Sci. 1989, 14, 97.

[26] Frey, P. A.; Sammons, R. D. Science 1985, 228, 541.

[27] Wilkins, E.; Carter, M.; Voss, J.; Ivnitski, D. Electrochem. Commun. 2000, 2, 786.

[28] Chen, P. S.; Huang, S. D. Talanta 2006, 69, 669.

[29] Salm, P.; Taylor, P. J.; Roberts, D.; Silva, J. D. J. Chromatogr. B 2009, 877, 568.

[30] You, Z.-S.; Wen, Y.-J.; Jiang, K.-Z.; Pan, Y.-J. Chin. Sci. Bull. 2012, 57,1183 . (尤珠双，文永均，蒋可志，潘远江，科学通报, 2012, 57, 1183.) 
[31] Zhang, J.; Chai, Y. F.; Wang, W.; Shang, W.; Pan, Y. J. Chinese J. Chem. 2012, 30, 2383.

[32] Chai, Y.-F.; Gan, S.-F.; Pan, Y.-J. Acta Chim. Sinica 2012, 70, 1805. (柴云峰，甘世凤，潘远江，化学学报, 2012, 70, 1805.)

[33] Yin, X.-C.; Jiang, Y.; Chu, S.-Y.; Weng, G.-F.; Fang, X.; Pan, Y.-J.
Acta Chim. Sinica 2018, 76, 436. (尹欣驰, 江游, 楚士颖, 翁国锋, 方向, 潘远江, 化学学报, 2018, 76, 436.)

[34] Zhang, X. P.; Chen, H. H.; Ji, Y.; Jiang, K. Z.; Chen, H. W. J. Am. Soc. Mass Spectrom. 2018, doi.org/10.1007/s13361-018-2098-4.

[35] Newman, M. S.; Karnes, H. A. J. Org. Chem. 1966, 31, 3980.

(Cheng, B.) 